\title{
Nursing Education Think Tank 2002
}

\section{Executive Summary}

Over the past decade, a growing body of evidence has highlighted the pivotal role of nursing education in meeting the future challenges in healthcare globally, particularly in addressing the solutions and challenges related to healthcare reform. In view of the importance of this issue, nursing leaders from government, education, research and healthcare and professional organizations gathered at the Nursing Education Think Tank 2002 to continue dialogue and determine approaches that will effectively shape the future of nursing education. The Nursing Education Think Tank 2002 was hosted by the Registered Nurses Association of Ontario (RNAO) in partnership with the Office of Nursing Policy, Health Canada (ONP-HC) and the Nursing Secretariat-Ontario Ministry of Health and Long-Term Care (MOHLTC). The Think Tank reflects the shared commitment to support coordinated planning and development of initiatives that will enable nurse educators and other nurse leaders to address the challenges of creating a dynamic system of nursing education as we move into the future.

In a review of six national and provincial nursing reports, the Think Tank Planning Committee identified key recommendations relevant to nursing education. Themes emerging from this review were identified as priority issues in nursing entry to practice and graduate education and provided a framework to guide the Think Tank discussions. These priority themes were broadly categorized as: curriculum, supply of nurses, clinical education and faculty supply.

On October 23, 2002, over 30 national and provincial nursing leaders participated in the Think Tank held at RNAO. At the outset, a brief update regarding the status of the recommendations to be considered was presented, following which the group collectively undertook a gap analysis of the recommendations, and the themes identified. Participants grouped according to theme, were given the following guid- 
ing question: "What can the nursing profession/nursing education do to mobilize action for this priority in the next 18 to 24 months?" For each theme, participants prioritized activities related to the recommendations, developed action plans, and identified key responsibilities to be carried out over the next 6 to 24 months.

The following priority activities related to each theme were identified:

\section{Curriculum}

- Revise the CASN National Position Statement on Nursing Education Curriculum to promote innovation and diversity, and to respond to issues such as regional access, technology, healthcare reform, changes in the body of nursing knowledge, scope of practice, health and education policy, advances in health sciences, population health needs and international dynamics.

- Recommend that the CASN accreditation standards related to curriculum be altered to reflect the revised CASN National Position Statement on Nursing Education Curriculum.

- Obtain funding for a national nursing education research chair to focus on investigation of teaching-learning modalities for enhancing nursing education as a practice discipline, and on further development of the knowledge base related to learning to nurse.

\section{Supply of Nurses}

- Standardize tracking and trending methods and approaches of student and early career attrition at the local, provincial and national levels.

- Raise awareness of and utilize data on local, provincial and national population health needs in planning nursing human resources.

- Design nursing programs to meet local needs.

\section{Practice Education}

- Establish a nursing practice and learning consortium as a model to coordinate comprehensive practice placements for students and practising nurses.

- Recommend that CASN Accreditation Board redefine clinical education to include a broader definition of practice while maintaining realistic employer expectations regarding novice nursing practice.

\section{Faculty Supply}

- Determine and plan for more effective utilization of existing, interim and ideal faculty supply, given the realities of both academia and the healthcare system.

- Enhance development opportunities for current faculty related to teaching and scholarship.

- Increase faculty supply.

These activities will require the concerted and collaborative efforts of key stakeholders including professional and educational nursing associations, government representatives, nurse researchers and clinicians across health care sectors.

Full report at www.rnao.org/html/PDF/

Final_Nursing_Education_Think_Tank_2002_ Report.pdf 